\title{
Automated analysis of microplastics based on vibrational spectros- copy: Are we measuring the same metrics?
}

\author{
Mingtan Dong ${ }^{1 *}$, Zhenbing She ${ }^{2,4}$, Xiong Xiong ${ }^{3}$, Zejiao Luo ${ }^{1 * *}$ \\ 1 School of Environmental Studies, China University of Geosciences, Wuhan 430078, China \\ 2 State Key Laboratory of Biogeology and Environmental Geology, China University of Geosciences, Wuhan 430078, \\ China \\ 3 State Key Laboratory of Freshwater Ecology and Biotechnology, Institute of Hydrobiology, Chinese Academy of Sci- \\ ences, Wuhan 430072, China \\ 4 School of Earth Sciences, China University of Geosciences, Wuhan 430074, China
}

\begin{abstract}
The traditional manual analysis of microplastics has been criticized for its labor-intensive, inaccurate identification of very small microplastics (less than $10 \mu \mathrm{m}$ ), and the lack of uniformity between instrumentation techniques. There are already three automated analysis strategies for microplastics based on vibrational spectroscopy: laser direct infrared (LDIR)-based particle analysis, Raman-based particle analysis, and focal plane array-Fourier transform infrared (FPA-FTIR) imaging. We compared the performances of these strategies in terms of their quantification, detection limit, size measurement, and material identification accuracy and analysis speed by analyzing the same standard and environmental samples. Unfortunately, the automated analysis results are not consistent in terms of quantification and material identification. The number of particles smaller than $60 \mu \mathrm{m}$ recognized by Ramanbased particle analysis is far greater than that recognized by LDIR-based particle analysis. Raman-based particle analysis has a submicrometer detection limit but should not be used in the automated analysis of microplastics in environmental samples because of the strong fluorescence interference. LDIR-based particle analysis provides the fastest analysis speed, but we suggest using a reliable detection limit of approximately $60 \mu \mathrm{m}$ and manually cross-check between the material identification results and the reference database used. Misidentification could occur due to the narrow tuning range from $1800-975 \mathrm{~cm}^{-1}$ and dispersive artefact distortion of infrared spectra collected in reflection mode. FPA-FTIR imaging provides relatively reliable quantification and material identification for microplastics in environmental samples greater than $20 \mu \mathrm{m}$ but might provide an imprecise description of the particle shapes. Optical photothermal infrared (O-PTIR) spectroscopy can detect submicron-sized environmental microplastics $(0.5-5 \mu \mathrm{m})$ intermingled with a substantial amount of biological matrix; the resulting spectra are searchable in infrared databases without the influence of fluorescence interference, but the process would need to be fully automated.
\end{abstract}

\section{INTRODUCTION}

It is almost certain that microplastics, plastics smaller than 5 $\mathrm{mm}^{1}$, exist in every environment of Earth's surface ${ }^{2}$, and all species may be exposed to microplastics ${ }^{1,3}$. Microplastics are emerging pollutants known for their interaction with other pollutants ${ }^{4}$ and potential toxicity to organisms ${ }^{5}$. Therefore, the accurate and efficient analysis of microplastics in different matrices represents a critical first step in assessing and describing the microplastic pollution. The entire analysis process of microplastics includes sampling, pretreatment, identification and quantification, but the 'analysis' in this study is specifically defined as the identification and quantification of microplastics.

Manual analysis of microplastics requires an operator to visually recognize, count, and measure microplastics under a stereomicroscope. Then, these suspected microplastics will be transferred into Fourier transform infrared (FTIR) spectroscopy, Raman spectroscopy, or pyrolysis-gas chromatography-mass spectrometry (Pyr-GC-MS) instruments to identify each their polymer type ${ }^{6}$. Particles smaller than $100 \mu \mathrm{m}$ are challenging to recognize visually ${ }^{7}$ and transfer into an instrument to identify their material types. Nile red staining can assist visual recognition of microplastics but cannot identify polymer types ${ }^{8}$. In addition, most studies consider only a part of the visually recognized microplastics to identify their polymer types ${ }^{9,10}$, but only $1.4 \%$ of suspected microplastics identified visually were confirmed as polymers ${ }^{11}$. This overall process is labor intensive, potentially inaccurate, easily affected by operator-related factors, and the rest of particles less than 100 um remain unidentified. Unfortunately, these smaller microplastics usually have a higher abundance in the environment ${ }^{2}$ and possess stronger toxicological effects ${ }^{12}$.

We define the term 'automated analysis of microplastics' as a strategy that could automatically recognize, count, measure the size of, and identify the material types of microplastics to quantify them. Such an approach should have low detection limits and be labor-free, efficient, and accurate. Although technologies such as secondary ion mass spectrometry (SIMS) ${ }^{13}$ and single particle-inductively coupled plasma mass spectrometry (SP-ICP-MS) ${ }^{14}$ have been applied for detecting microplastics in recent years, they are more challenging to adapt for environmental samples ${ }^{14}$. In contrast, the automated analysis of microplastics based on vibrational spectroscopy (both Raman and infrared) is more compatible with existing studies and sample pretreatment methods. There exist three strategies for the automated analysis of microplastics: laser direct infrared (LDIR)-based particle analysis ${ }^{15}$, Raman-based particle analysis ${ }^{16}$, and focal plane array (FPA)-FTIR imaging ${ }^{2,17-19}$. They are based on different instruments and principles. Quantitative results from manual analyses of microplastics are difficult to compare between different research groups ${ }^{20-22}$. Naturally, a 
new question arises: Are we measuring the same metrics among these automated microplastics analysis strategies?

Therefore, for technique validation, we designed a comparative study of the performance of these strategies in the automated analysis of microplastics. The same standard microplastic sample and environmental microplastic sample were analyzed with different strategies. The comparative targets included the detection limit, size measurement, material identification, quantification results, and analysis speed. Additionally, the performances of optical photothermal infrared (O-PTIR) spectroscopy ${ }^{23,24}$ and Raman mapping (Raman imaging) ${ }^{25,26}$ were compared with that of Raman-based particle analysis for detecting microplastics smaller than $5 \mu \mathrm{m}$. The sample preparation requirements of different strategies were also discussed.

\section{EXPERIMENTAL SECTION}

\section{Microplastic sample preparation}

Eight virgin microplastic particle powders (Table S1) were purchased from Ante Plastic Materials Co., Ltd. (China). Different from the commonly used polystyrene microspheres ${ }^{27}$, these standard microplastic powders are irregular particles, including polyethylene (PE), polyethylene terephthalate (PET), polyvinyl chloride (PVC), polyamide (PA), and polystyrene (PS), with particle sizes ranging from approximately $1 \mu \mathrm{m}$ to $300 \mu \mathrm{m}$. Then, these different microplastic powders with different polymer types and sizes were independently suspended in absolute ethyl alcohol to be used as the standard microplastic sample (Fig. 1).

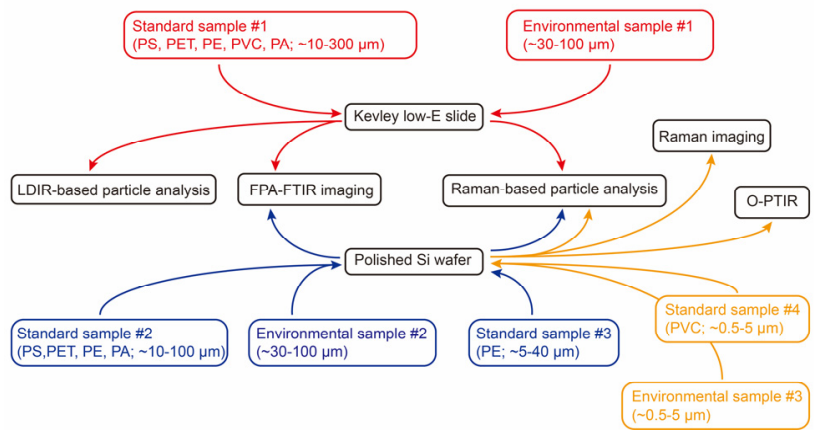

Figure 1. Flow chart of the comparative study between different strategies for the automated analysis of microplastics.

Environmental microplastics (Fig. S1) were extracted with $\mathrm{ZnCl}_{2}\left(1.7 \mathrm{~g} \cdot \mathrm{cm}^{-3}\right)$ from the soil of one of the world's largest plastic waste recycling bases ${ }^{28}$. The environmental microplastics were filtered through 150 mesh, 500 mesh, and 3000 mesh metal sieve stacks. Environmental microplastics with a size of approximately $30-100 \mu \mathrm{m}$ were rinsed from the sieve with absolute ethyl alcohol and used as the environmental samples \#1 and \#2 (Fig. 1). Environmental microplastics smaller than $5 \mu \mathrm{m}$ were filtered onto a membrane (polyethersulfone, pore $0.45 \mu \mathrm{m}$, Supor-450, Pall Corporation), ultrasonic extracted with absolute ethyl alcohol, and used as environmental sample \#3 (Fig. $1)$.

\section{LDIR-based particle analysis}

Agilent 8700 LDIR (Agilent Solutions, Inc., USA) is a novel technique that illuminates samples with a mid-IR quantum cascade laser (QCL), which tunes from $1800-975 \mathrm{~cm}^{-1}$ at a spectral resolution of $0.5 \mathrm{~cm}^{-1}$, as the light source instead of FTIR's globar. Infrared light from the QCL is directed to the sample, scattered light would reflect off the sample, and would be subsequently directed to a thermoelectrically cooled mercury- cadmium-telluride (MCT) detector for signal processing. Agilent 8700 LDIR only accepts glass slides for loading samples. The particles dispersed in the ethanol solution are dropped onto Kevley low-E slides (Fig. S2a). First, the instrument obtains a bright image with a large-field-of-view camera (optical resolution of approximately $24 \mu \mathrm{m}$ ). Then, it uses single wavelength light $\left(1800 \mathrm{~cm}^{-1}\right)$ with a 0.72 numerical aperture (NA) objective for a theoretical spatial resolution of $4.7 \mu \mathrm{m}(0.61 * 10,000 / 1800$ $\mathrm{cm}^{-1} / 0.72$ ) to survey, count, locate and measure the particles in this image. Third, it measures the infrared spectrum of each particle in reflection mode. Finally, Clarity software (version 1.3.42, Agilent, USA) is used to automatically compare the spectrum with the reference spectral library to identify the material types. Spectra with a match quality smaller than 0.65 were listed as "Undefined.". The reference spectral library contains polymer and non-polymer materials (e.g., coal, alkyd varnish, and chitin). Selected optical images of the particles of interest can be acquired and studied in detail under the objective (visible resolution of $1 \mu \mathrm{m}$ ). In this work, the detection threshold was set to $20 \mu \mathrm{m}$. The optical image of the particles in environmental sample \#1 was re-acquired under the objective after regular analysis (to check for consistency).

\section{Raman-based particle analysis}

WITec ParticleScout (version 5.3.14.106) is software used with a WITec alpha300 R confocal Raman imaging system (WITec GmbH, Germany). First, it obtains a large-area image by the image-stitching and focus-stacking function. Then, it surveys, measures, and locates particles in this image. Next, it acquires the Raman spectrum of each particle with the Autofocus setting and identifies them by linking the WITec TrueMatch database. The logic of WITec ParticleScout in detecting microplastics is similar to that of Agilent 8700 LDIR.

The WITec alpha300 R spectroscope used was equipped with a $532 \mathrm{~nm}$ laser. The grating used had a groove density of 600 lines per millimeter and a Blaze wavelength (BLZ) of $500 \mathrm{~nm}$. The Raman shift ranged from $0-4000 \mathrm{~cm}^{-1}$. A 20x darkfield objective (EC Epiplan-Neofluar HD, Zeiss) with 0.5 NA was used in the analysis of standard samples \#1, \#2, and \#3 and environmental samples \#1 and \#2 (Fig. 1). A 100x bright field objective (EC Epiplan-Neofluar Dic, Zeiss) with 0.9 NA was used to analyze standard sample \#4 and environmental samples \#3 (Fig. 1). The pixel sizes of visible light images taken under the $20 \times$ and $100 \times$ objectives were $0.61 \mu \mathrm{m}$ and $0.12 \mu \mathrm{m}$, respectively. According to the Rayleigh criterion, the lateral resolution $(0.61 \lambda / \mathrm{NA})$ of the Raman spectra acquired under the $20 \times$ and $100 \times$ objectives were $0.65 \mu \mathrm{m}$ and $0.36 \mu \mathrm{m}$, respectively, and the axial resolutions in the air $\left(1.4 \lambda / \mathrm{NA}^{2}\right)$ were $3 \mu \mathrm{m}$ and 0.92 $\mu \mathrm{m}$, respectively. The vignetting of the image was corrected in real time to prevent it from affecting particle recognition. The threshold of finding particles from the dark field images was automatically set by ParticleScout. The spectrum of each particle was automatically acquired with signal optimization settings (integration time: $1 \mathrm{~s}$; accumulation number: 3 or 5 times; and laser power: $5 \mathrm{~mW}$ ). Autofocus setting was performed for the Raman shift range of $2800-3200 \mathrm{~cm}^{-1}$, and the steps under the $20 \times$ and $100 \times$ objectives were set to $2 \mu \mathrm{m}$ and $0.2 \mu \mathrm{m}$, respectively. The Raman Database of Weathered Microplastics built in our previous study ${ }^{28}$ and a commercial polymer database (ST Japan 5.2) containing 4568 Raman spectra were used to confirm polymer types through WITec TrueMatch Database software. Spectra with a hit index quality (HQI) smaller than 60 were listed as 'Unknown.' In addition, the Raman mapping performed to detect the microplastics in standard sample \#4 followed the same Raman spectroscopy approach. ParticleScout 
was also used to count and measure particles from the Raman map.

Additionally, Raman spectra are typically affected by autofluorescence interference ${ }^{28,29}$. Another two WITec alpha300 R instruments equipped with 488 and $785 \mathrm{~nm}$ lasers were used to measure the Raman spectra of some particles in the environmental samples to compare the performance of different lasers in reducing fluorescence interference.

\section{FPA-FTIR imaging and data process}

A LUMOS II FT-IR imaging microscope (Bruker Optics $\mathrm{GmbH}$, Germany) equipped with $32 \times 32$ Focal-Plane Array (FPA) detectors and can acquire 1024 infrared spectra in a single scan at a minimum spatial resolution of $5 \mu \mathrm{m}$. The logic of FPA-FTIR imaging in detecting microplastics is different from the particle recognition-spectrum measurement mode of particle analysis. FPA-FTIR spectroscopy detects microplastics from hyperspectral chemical imaging rather than visible light imaging or single-wave imaging. As early as 2015, FPA-FTIR imaging was used to identify microplastics from environmental matrix ${ }^{11,18,30}$. However, the visualization of microplastics based on the integration of a single peak cannot cope with different polymer types, and the considerable amount of data makes this approach unable to be widely used in the automated analysis of microplastics ${ }^{31}$. Within the past two years, multivariate statistics, data mining, and machine learning methods, such as principal component analysis ${ }^{32}$, cluster analysis ${ }^{32}$, random decision forest (RDF) classifiers ${ }^{33}$, and partial least squares discriminant analysis ${ }^{17}$, were applied to process hyperspectral imaging. These methods make it possible to reduce the dimensionality of hyperspectral infrared imaging and detect and quantify microplastics. Two software programs, siMPle developed by Primpke et al. ${ }^{34}$ and Purency Microplastics Finder (Purency GmbH, Austria), can be applied in processing FPA-FTIR imaging.

In this work, the FPA detectors measured the infrared spectra in reflection mode with a spectral resolution of $8 \mathrm{~cm}^{-1}$ and 2 scans. The wavenumber ranged from $4000-750 \mathrm{~cm}^{-1}$. Except for standard sample \#3 (Fig. 1), which was measured at a spatial resolution of $5 \mu \mathrm{m}$ (no binning), the other samples (Fig. 1) were measured at a spatial resolution of $10 \mu \mathrm{m}(2 \times 2$ binning). The background was measured with the same parameters and was subtracted from the results. Then, the hyperspectral data were imported into Purency Microplastics Finder (version 3.49) running with a machine learning model (version PMF_R2021a) based on the RDF classifiers ${ }^{33}$ to detect, measure, and classify the microplastics. The spectra from $3600-1250 \mathrm{~cm}^{-1}$ were used for the Purency calculations. This process only detected the microplastics, and other particles were listed as 'Unknown.'

The machine learning model in Purency Microplastics Finder was trained with the transmission spectrum imaging data obtained with an aluminum oxide filter ${ }^{33}$. Although the Kevley low-E slides were designed for reflective infrared measurements and were used in the LDIR spectral measurements, the infrared spectra of the Kevley low-E slides (Fig. S3b) differed from the infrared spectra of aluminum oxide (Fig. S3a) and were not compatible for the Purency Microplastics Finder. Therefore, the edges of the microplastics loaded on the Kevley low-E slide are poorly recognized (Fig. S5). The quantification and size measurement of standard sample \#1 and environmental sample \#1 acquired by the Bruker-Purency Microplastics Finder strategy could not be compared with those acquired by the LDIR-based or Raman-based particle analysis; only the results of the material identification and operational speed were compared. Another three samples were loaded on a polished silicon wafer (Fig. S2b) to compare the quantification and size measurement results between the FPA-FTIR imaging and Raman-based particle analysis strategies (Fig. 1). Reflection mode was used to maintain consistency between these methodologies within this study. We noticed that the FTIR reflection spectrum of the polished silicon wafer had a different band shape in the polyethylene particles and a smaller absorption baseline than the transmission spectrum (Fig. S4).

Note that the FPA-FTIR imaging strategy was not run under the optimal settings to meet the sample preparation requirements of different strategies, which may adversely affect the results.

\section{O-PTIR measurements}

O-PTIR spectra and imaging was measured on a commercial instrument, mIRage $+\mathrm{R}$ (Photothermal Spectroscopy Corp., USA). The system is equipped a QCL with the wavenumber ranged from $1800-800 \mathrm{~cm}^{-1}$, but the principles and working mode of O-PTIR are different from QCL-IR (LDIR). The OPTIR technique can be described as the "pump-probe" technique, whereby the QCL infrared laser (the pump) is used to excite the sample and induce a "photothermal IR effect," where slight sample expansion and refractive index changes occur, which are probed in reflection mode via a visible laser beam $(532 \mathrm{~nm})$. The intensity change of the visible light is proportional to the absorption of infrared radiation ${ }^{35,36}$. O-PTIR uses a visible light probe to detect the photothermal event induced by the incident infrared radiation ${ }^{35,36}$. The O-PTIR instrument was equipped with a $532 \mathrm{~nm}$ laser as the probe and a $40 \times$ objective $(0.78 \mathrm{NA})$, so the theoretical resolution of O-PTIR is approximately $500 \mathrm{~nm}$. The system is also equipped with an iHR320 imaging spectrometer (Horiba, Japan) and could simultaneously acquire Raman spectrum excited by the $532 \mathrm{~nm}$ laser at the same time, same spot, and same resolution during the measurement of O-PTIR spectra.

The O-PTIR instrument was employed to measure the OPTIR and Raman spectra of standard PVC particles ranged from about 0.5-5 $\mu \mathrm{m}$ and environmental microplastic sample \#3 (Fig. $1)$. The IR power, probe power, scan rate, and step size were adjusted and optimized based on-demand. O-PTIR spectra can be directly transferred into KnowItAll Information System (Wiley, USA) for spectral matching and deconvolution analysis.

\section{Quality assurance and quality control}

The LDIR-based particle analysis strategy analyzed the samples first. Then, the samples were stored in separate hermetic boxes and were transported to different laboratories in-person to avoid vibration and sample damage by third-party carriers. Each comparison group (Fig. 1) was completed within one week to ensure the consistency of the particles. The area of Raman-based particle analysis and FPA-FTIR imaging were slightly larger than those of the LDIR-based particle analysis, which made it easier to compare particles. Particles were crosschecked in terms of their position and shape to compare the size measurement and material identification results for the same particle. Two particle size standards of $10.12 \pm 0.06 \mu \mathrm{m}(4 \mathrm{~K}-10$, Duke Standards, Thermo Scientific, USA) and $100 \pm 1.5 \mu \mathrm{m}$ (4K-100, Duke Standards, Thermo Scientific, USA) were used to validate the size measurement results of the Raman-based particle analysis.

\section{RESULTS AND DISCUSSION}

\section{Quantification and detection limits}

Since manta and neuston nets are often used in the sampling of microplastics and metal sieves are used for prefiltration ${ }^{6,22}$, 
the width of the particles is used as the size. The number of particles smaller than $60 \mu \mathrm{m}$ recognized by the LDIR-based particle analysis is much smaller than that recognized by the Raman-based particle analysis (Fig. 2a, c), which means the LDIR-based particle analysis is not accurate in the quantification of microplastics smaller than $60 \mu \mathrm{m}$. Thus, the percentage of microplastics smaller than $60 \mu \mathrm{m}$ is expected to be underestimated in studies using LDIR-based particle analysis ${ }^{15,37,38}$. The Raman-based particle analysis is based on darkfield images with a resolution of $0.61 \mu \mathrm{m}$, which helps recognize very small particles. For standard sample \#1, the quantitative results of microplastics under Raman-based particle analysis are much higher than those under LDIR-based particle analysis (Fig. 2b). For environmental sample \#1, the number of microplastics detected by Raman-based particle analysis is smaller than that detected by LDIR-based particle analysis (Fig. 2d) because of the differences in material identification, which then affects the quantification of the microplastics.

In standard sample \#2, the microplastics smaller than $30 \mu \mathrm{m}$ quantified by Raman-based particle analysis are much more abundant than those quantified by FPA-FTIR imaging (Fig. 2e) due to $2 \times 2$ binning for a $10 \mu \mathrm{m}$ pixel resolution. In standard sample \#3, the microplastics quantified by FPA-FTIR imaging with a $5 \mu \mathrm{m}$-pixel resolution (no binning) are more similar to those quantified by Raman-based particle analysis (Fig. 2f). However, the number of microplastics in environmental sample \#2 detected by Raman-based particle analysis is much lower than that detected by FPA-FTIR imaging (Fig. 2g), similar to the difference observed for environmental sample \#1 between the LDIR-based particle analysis and Raman-based particle analysis results (Fig. 2d).

The difference in the quantitative results is affected by the detection limit, particle recognition, size measurement, and material identification of the microplastics. Different strategies have different detection limits (Fig. 2h). We do not suggest using the lowest detection limit to quantify microplastics because these strategies are often inaccurate for quantifying microplastics close to the detection limit (Fig. 2a, c). We recommend using a more reliable detection limit based on the consideration of the spatial resolution of the spectroscopic technique (Fig. 2h). (a) Standard sample \#1 particles

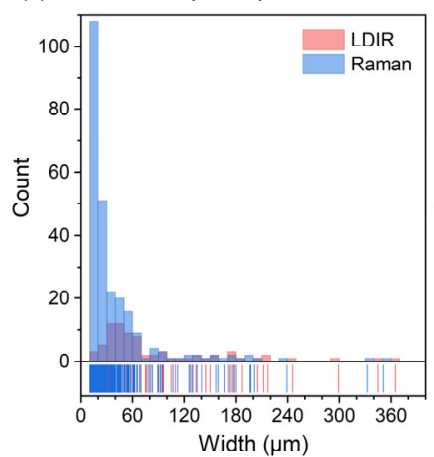

(e) Standard sample \#2 microplastics

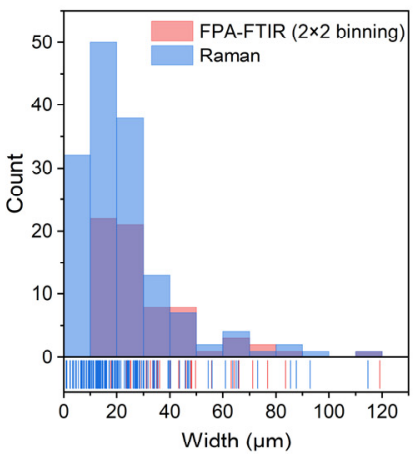

(b) Standard sample \#1 microplastics

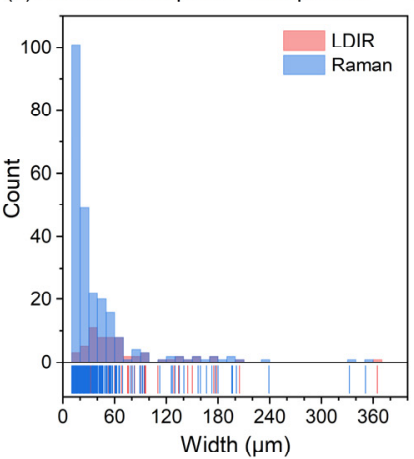

(f) Standard sample \#3 microplastics

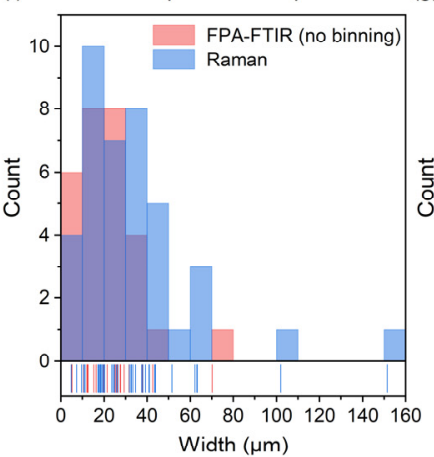

(c) Environmental sample \#1 particles

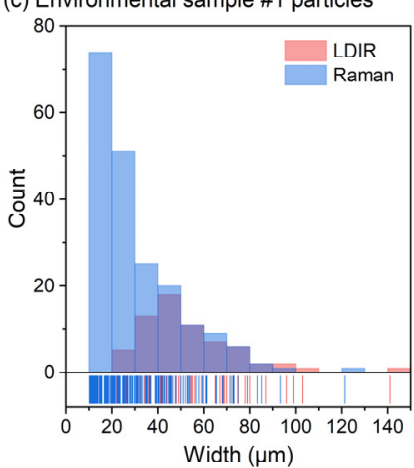

(g) Environmental sample \#2 microplastics

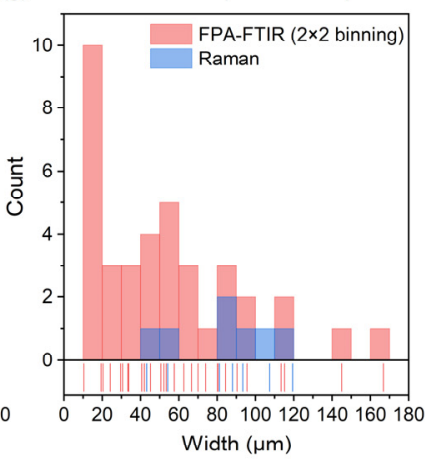

(d) Environmental sample \#1 microplastics

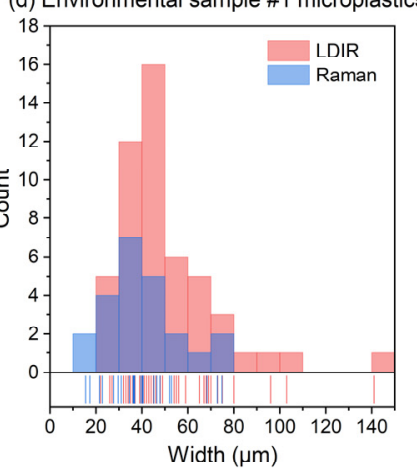

(h) Detection limits

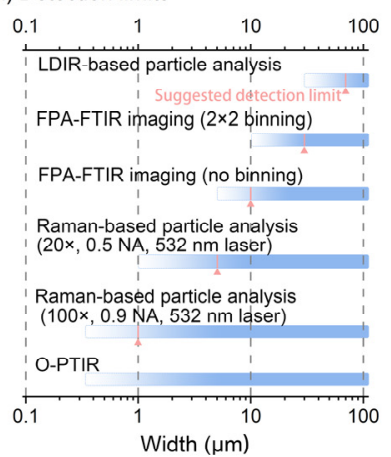

Figure 2. Quantification results of different strategies for the same sample (a-g) and the detection limits of different strategies (h).

\section{Size measurement}

The measurement result of the Raman-based particle analysis for the spherical PS standard with a particle size of $10.12 \pm 0.06$ $\mu \mathrm{m}$ is $12.23 \pm 0.74 \mu \mathrm{m}$, and the measurement result for the standard with a particle size of $100 \pm 1.5 \mu \mathrm{m}$ is $97.68 \pm 4.46 \mu \mathrm{m}$. It is difficult to find the equatorial plane of spherical particles. The particle roughness may influence edge recognition in creating a particle mask. Generally, the size measurement of the Ramanbased particle analysis is accurate. The size measurement of the LDIR-based particle analysis is linearly correlated to the Raman-based particle analysis (Fig. S6a, b). However, the slope of the linear fit is not 1 , and the intercept is not 0 , indicating a difference between the size measurements of Raman-based and LDIR-based particle analysis.

A gap persisted between the particle sizes measured by Raman-based particle analysis and FPA-FTIR imaging (Fig. S6c, $d, e)$. The particle sizes measured by FPA-FTIR imaging seem to be an order or magnitude greater than the resolution. For example, the particle sizes measured under $2 \times 2$ binning are often $10 \mu \mathrm{m}$ to $20 \mu \mathrm{m}$ (Fig. S6c). Clearly, the particle size measured with a resolution of $10 \mu \mathrm{m}$ is far less accurate than that measured by Raman-based particle analysis (Fig. 3a, d). Thus, a higher resolution corresponds to higher accuracy in the size measurement. In addition, the particle size measurement under 
FPA-FTIR imaging is also related to the recognition of particle edges. The uneven thickness, varying degrees of weathering, and uncontrolled association of naturally occurring exogenous matrix within one microplastic particle would affect the corresponding infrared spectra; thus, creating particle masked with Purency Microplastics Finder is challenging (Fig. 3b). Although Purency Microplastics Finder allowed the users to re-edit the particle mask to improve the accuracy of the particle measurement, this step would increase the workload and was not applied in this study. Raman-based particle analysis is not affected by the inconsistent spectra of the particles themselves (Fig. 3e), but FPA-FTIR had an advantage for distinguishing adjacent particles with different polymer types (Fig. 3c), which also could be recognized by analyzing one microplastic particle at a time (Fig. 3f).

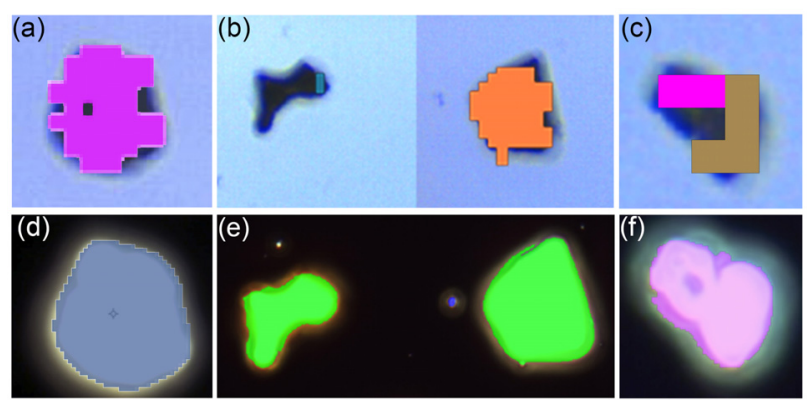

Figure 3. Microplastic masks created by FPA-FTIR imaging (a, b, c) and Raman-based particle analysis (d, e, f). (a, c, d and f) are from the material maps of standard sample \#2 (Figs. S8 and S9), but (b) and (e) are from environmental sample \#2. The different colors in $(\mathrm{a}, \mathrm{b}$, and $\mathrm{c})$ represent different polymer types.

\section{Material identification}

Raman-based particle analysis and FPA-FTIR imaging are consistent in identifying particles in standard sample \#1, but they are inconsistent with LDIR-based particle analysis (Fig. 4a). In the LDIR-based particle analysis, PET may be erroneously classified as alkyd varnish, polyurethane (PU), PA, and rubber, and PA is erroneously classified as chitin (Fig. 4a). In fact, both the LDIR and FTIR spectra are demonstrating band shapes consistent with spectral artefacts associated with the dispersive scatter artefacts (Mie Scatter) ${ }^{39}$ (Fig. 4c, d), where when the particles sizes are in the order of the wavelengths of light being used to measure, spectra will be significantly distorted with baseline shifts/slopes, peak shifts/splitting and band ratio changes. This effect, though also present in transmission mode, is most acute when spectra are collected in reflection mode. The issue is significant as spectral profile is no longer representative of the chemistry of the sample, but is in fact much more heavily dominated by the dispersive scattering artefacts that depend on particle shape and size. In other words, spectral reproducibility is significantly negatively impacted with "direct" IR techniques like LDIR and also FTIR. These dispersive scatter issues are not present in Raman and O-PTIR. The incorrect classification of particles is also related to the built-in database of Clarity. Clarity's built-in database includes nonplastic materials such as chitin and alkyd varnish, resulting in overmatching phenomena in standard sample \#1. When the nonplastic materials were removed from the library, the revised material identification results of the LDIR-based particle analysis strategy were closer to those of Raman-based particle analysis and FPA-FTIR imaging. In addition, the wavenumber range of the LDIR-based particle analysis is only $1800-975 \mathrm{~cm}^{-}$ 1 , restricting the polymer identification from referring to the stretching vibration of C-H bonds at $3000-2800 \mathrm{~cm}^{-1}$. The FPA-
FTIR imaging strategy has a wider wavenumber range, 3600$1250 \mathrm{~cm}^{-1}$, and Raman-based particle analysis covers the range of $0-4000 \mathrm{~cm}^{-1}$, making material identification more definite.

The difference in material identification of particles in environmental samples between these three strategies is even greater (Fig. 4b). The LDIR- and FTIR-based identification of polypropylene (PP), PE and PU are basically consistent, but the LDIR classifies acrylonitrile butadiene styrene (ABS) as PS because of the stretching vibration of $\mathrm{C} \equiv \mathrm{N}$ in $\mathrm{ABS}$ located at $2236 \mathrm{~cm}^{-1}$, beyond $1800-975 \mathrm{~cm}^{-1}$ (Fig. 4e). There will be a series of changes in the infrared spectrum of weathered microplastics, such as a C-O stretching vibration peak at $1010 \mathrm{~cm}^{-1}$, a $\mathrm{C}=\mathrm{C}$ stretching vibration peak at $1640 \mathrm{~cm}^{-1}$, and a series of carbonyl stretching vibration peaks at $1800-1680 \mathrm{~cm}^{-128}$. In contrast, the peaks of the C-H stretching vibration of 3000-2800 $\mathrm{cm}^{-1}$ of polymers are more robust against weathering ${ }^{28}$.

The Raman spectra of weathered microplastics ${ }^{7,28}$ and microplastics with pigment additives ${ }^{29}$ often have strong fluorescence and cannot be identified (Fig. 4e). Switching lasers usually reduces fluorescence interference, but a single laser cannot cope with the diversity of particles in environmental samples in particle analysis mode. For some particles, 488, 532, and 785 nm lasers cannot obtain effective Raman spectra (Fig. S7d-g), which means that Raman-based particle analysis is not reliable in quantifying microplastics in environmental samples (Fig. 2g). For some other particles, the use of a $785 \mathrm{~nm}$ laser may help to weaken the fluorescence (Fig. S7a, h, j). However, the chargecoupled-device (CCD) detector responds poorly to the Raman shift beyond $2000 \mathrm{~cm}^{-1}$ excited by $785 \mathrm{~nm}^{40}$, making it challenging to identify polymers without $\mathrm{C}-\mathrm{H}$ stretching vibrations at $3200-2800 \mathrm{~cm}^{-1}$ (Fig. S7b, c, j). In addition, unlike the Raman shift between $2800-3200 \mathrm{~cm}^{-1}$, which could be selected as the range of autofocus when using a 488 or $532 \mathrm{~nm}$ laser, the complete Raman shift can be selected as the range of autofocus, which makes it easy to focus on the glass slide or substrate instead of the particles (Fig. S7c, i, j). Therefore, a $785 \mathrm{~nm}$ laser is not recommended in the Raman-based automated analysis of microplastics. Although non-plastic materials are not the focus of this study, we find that silicone in the LDIR-based particle analysis is identified as a carbonate mineral by Raman-based particle analysis (Fig. S10). Still, Raman-based particle analysis should not be used for the automated analysis of microplastics in environmental samples based on the consideration of strong fluorescence interference even with the $785 \mathrm{~nm}$ excitation laser source; but it may be possible to use this method to quantify the release of microplastics and nanoplastics in daily products ${ }^{41,42}$.

Reference databases are crucial in material identification. After decades of development of FTIR and Raman technologies, reference databases for a variety of polymers and polymer additives have been accumulated mostly in ideal measurement conditions, e.g. flat film or dilute dispersion in $\mathrm{KBr}$ pellet measured in FTIR transmission mode or Raman reflection mode. However, when identifying microplastics in environmental samples, it is best to use microplastics with environmental characteristics to create a database to address the influence of weathering on FTIR or Raman spectra ${ }^{7}$, such as those included in the Spectral Library of Plastic Particles Aged in the Environment (SLoPP-E) ${ }^{43}$, the Raman Database of Weathered Microplastics (RDWP) ${ }^{28}$ and the FTIR reference database designed for the automated analysis of microplastics ${ }^{44}$. Recently, Open Specy software has been developed for allowing researchers to share, view, process, and identify their spectra for free ${ }^{45}$. In addition, the sharp edges of microplastic fragments will strongly alter infrared spectral shapes due to the scattering of infrared lights that 
would lead to dispersive artefacts in the LDIR ad FTIR spectra (Fig. 4c, d, e; Fig. 5f, g) ${ }^{39}$. Changes in the spectral shapes will be dependent on the particle size as well, potentially leading to misidentification of microplastic fragments with these "direct" methods. LDIR-based particle analysis and FTIR in reflection mode are not directly compatible with FTIR transmission mode spectrum due to the inherent spectral artefact from its reflection mode operation; for LDIR, spectral processing will need to be revised in Clarity (version 1.3.42).

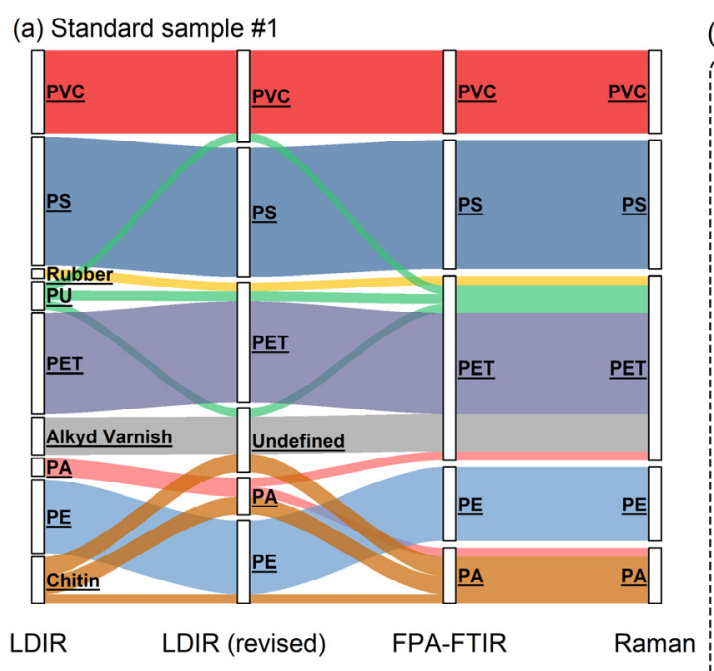

(c) 52-339-96 Standard sample \#1 11-164-213

(b) Environmental sample \#1
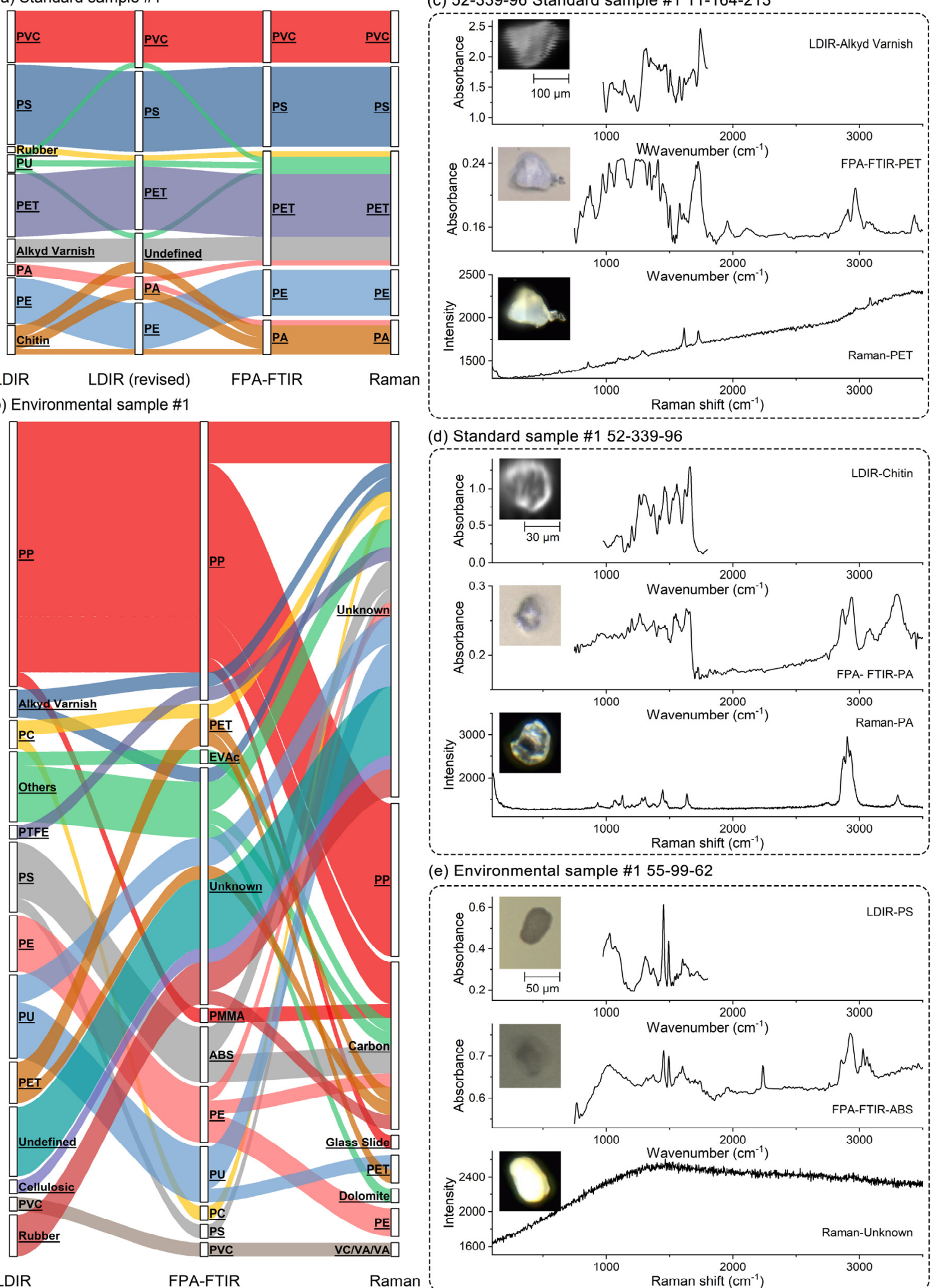

(d) Standard sample \#1 52-339-96

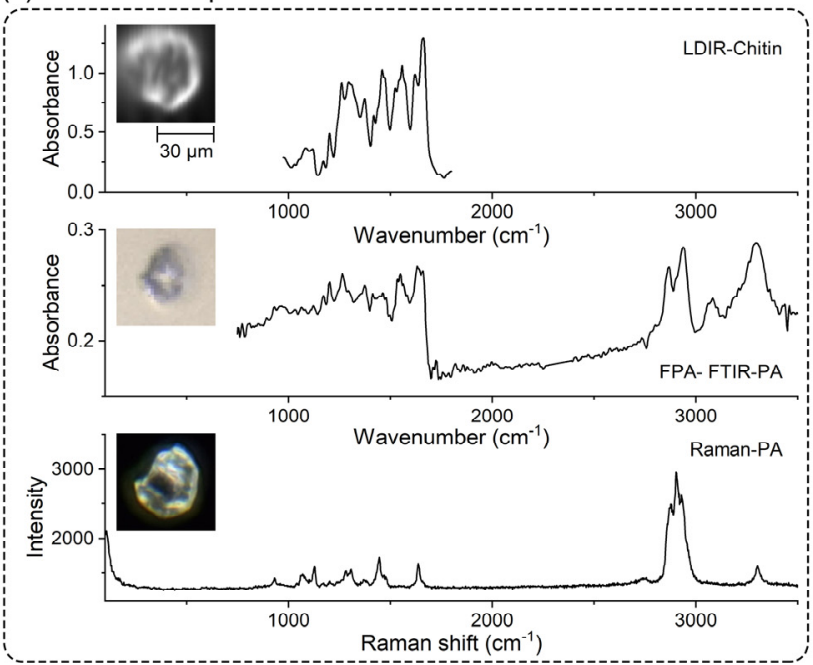

(e) Environmental sample \#1 55-99-62

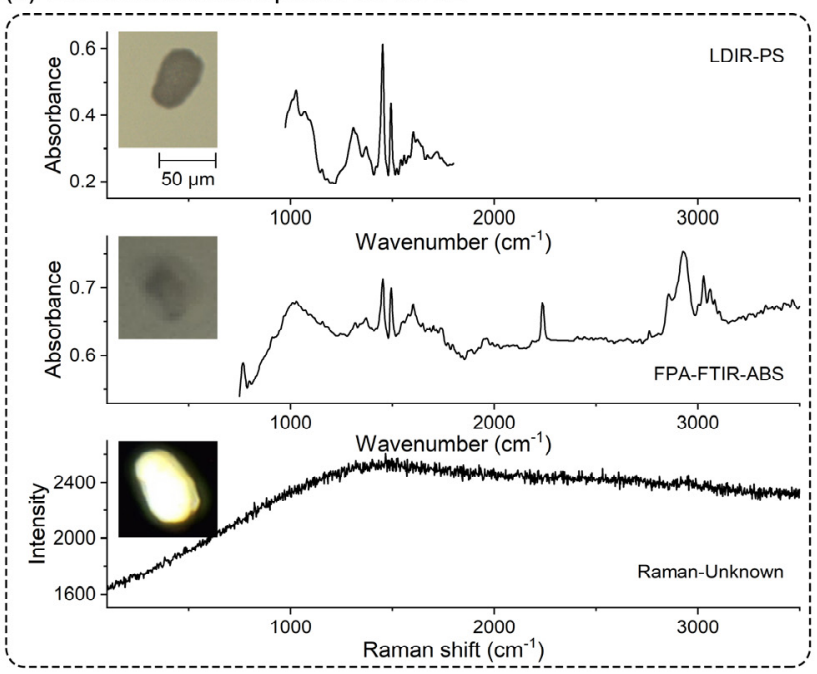

Figure 4. Comparison of the material identification results of standard sample \#1 (a) and environmental sample \#1 (b) under different strategies. LDIR, FTIR and Raman spectra for the same particle (c, d, e). 


\section{Speed}

The LDIR-based, O-PTIR-based, and Raman-based particle analysis are based on point analysis, henceforth the average time required for measuring a single particle can be calculated and compared (Table S2). It is meaningless to compare the total times required because the number of particles detected differs (Fig. 2). The average measurement time per particle under the LDIR-based particle analysis is approximately 6-9 s, while the average measurement time per particle under the Raman-based particle analysis is $14-15 \mathrm{~s}$. The time used by the Raman-based particle analysis is related to the integration time, cumulation time, autofocus range and steps. The acquisition time for each simultaneous O-PTIR and Raman spectral pair is in the range of $2-40$ seconds depending on the particle size.

Only the time required for measuring a certain area $\left(1 \mathrm{~mm}^{2}\right)$ can be calculated for FPA-FTIR imaging. It takes approximately 2 min to measure $1 \mathrm{~mm}^{2}$ with the $2 \times 2$ binning setting (resolution of $10 \mu \mathrm{m}$ ) and approximately $9 \mathrm{~min}$ to measure 1 $\mathrm{mm}^{2}$ without binning (pixel resolution of $5 \mu \mathrm{m}$ ), approximately 4 times that with $2 \times 2$ binning. It takes approximately 7 hours to measure an entire aluminum oxide filter $(14.9 \times 15.5 \mathrm{~mm}, 4 \times 4$ binning, Fig. S11). If there are many particles in a small area, FPA-FTIR imaging will be preferred. If there are a few particles in a large area, then particle analysis will be preferred.

\section{Submicron sized microplastics $(0.5-5 \mu \mathrm{m})$}

The Raman mapping (Fig. 5c) and O-PTIR image (Fig. 5d) of PVC particles (Fig. 5a) are the same as the material map created by Raman-based particle analysis (Fig. 5b). Specifically, Raman mapping provides a more detailed description of the edges of particles (Fig. 5c), while Raman-based particle analysis provides a smoother edge measurement (Fig. 5b). Therefore, the particle size measured with Raman-based particle analysis is larger than that measured by Raman mapping. For example, two adjacent microplastic particles (Fig. S12c) were not successfully separated by Raman-based particle analysis (Fig. $\mathrm{S} 12 \mathrm{~b}$ ), but signal isolation was successful by Raman mapping (Fig. S12a). The smallest microplastics detected by Ramanbased particle analysis is $0.61 \mu \mathrm{m}$ (Fig. S12b). Raman mapping and O-PTIR imaging can locate microplastic fragments of sizes above $0.5 \mu \mathrm{m}$ (Fig. S13). A high-precision motor stage can be used to over-sample for the resolution of the Raman spectrum to detect nanoplastics with a size of $30 \mathrm{~nm}^{26,46,47}$.

O-PTIR microspectroscopy can be used to analyze environmental microplastics smaller than $5 \mu \mathrm{m}$ without the effects of fluorescence interference (Fig. 5j, k). Similar to LDIR, O-PTIR spectroscopy also illuminates the sample with a tunable infrared source going from $1800-800 \mathrm{~cm}^{-1}$; but the infrared absorption is detected with a second probe laser beam in a pump-probe arrangement rather than measuring the reflected infrared light from the sample surface in the LDIR. In spite of its reflection mode spectral collection setup, the natural band shape of the O-PTIR spectra has no distortion and is closely resembling conventional FTIR in transmission mode (Fig. 5e). Additionally, the lack of dispersive scatter artefact susceptibility means that O-PTIR spectra are reproducible, despite differences in particle shape and size. These key features thus allow direct spectral searches in commercially available databases, which are comprised of infrared spectra collected in either transmission and corrected ATR modes, without custom library development.

O-PTIR spectra SP02 and SP03 have been collected from the same particle (Fig. 5i), but only SP02 shows distinctive spectroscopic differences around 1493 and $1452 \mathrm{~cm}^{-1}$ (Fig. 5j). A background material might ubiquitously exist (Fig. S13). With Wiley KnowItAll's deconvolution algorithm, two infrared absorption bands could be de-mixed from the matrix and are clearly consistent with a polystyrene derivative: the sharp $1493 \mathrm{~cm}^{-1}$ for $\mathrm{C}=\mathrm{C}$ stretch and a slightly wider $1452 \mathrm{~cm}^{-1}\left(\mathrm{C}=\mathrm{C}\right.$ and $\mathrm{CH}_{2}$ scissor in the backbone). For environmental samples, these microplastics will have microbes associated with them along with humic acids and other matters ${ }^{28,48,49}$ - all would muddle the spectra of the microplastics fragments. The strong broad $1590 \mathrm{~cm}^{-1}$ and the $1734 \mathrm{~cm}^{-1}$ show up very often and they are considered to be the background matter (Fig. $\mathrm{S} 14 \mathrm{~b}$ ). The former is consistent with some forms of humic acids 50 and the latter could be naturally occurring fats and esters. Therefore, it is very important to have good band shapes in the infrared spectra so that we can deconvolve the mixture spectrum into probable microplastic components with commercially available infrared databases, which are typically constructed based on transmission or ATR mode infrared spectra to enable the full capability of identifying unknown matters. As a result, smaller particles will take longer to analyze, not just because the actual field of view (FOV) is smaller but also because of their association to naturally occurring matters ${ }^{50-52}$. The combination of normal spectral shapes, high spatial resolution and high sensitivity to small sampling volume enable detection of small microplastic fragments that have a high affinity for attracting non-polar environmental contamination ${ }^{53}$. These natural matters are typically difficult to remove to return these microplastics fragments to the condition like that of the standard PVC sample \#4. With the O-PTIR technique, such a stringent sample preparation process can be significantly relaxed. As eluded at the beginning, Raman spectroscopy could not be sufficiently analyzed due to the strong fluorescence baseline. Furthermore, future algorithms to analyze these smaller fragments will need to include not only automated particle identifiers for locating the particles; but also include some form of artificial intelligence to recognize some of these common "synthetic material" bands as part of the pattern recognition process. Infrared spectroscopic profiles consistent with background materials would also be necessary to improve on background material subtraction and also reducing over-interpretation of the same. The infrared source of the O-PTIR instrument can be extended into the high wavenumber region, namely $3600-2700$ $\mathrm{cm}^{-1} 54$, to further enhance the accuracy of microplastic identification. For the first time, small environmental microplastics less than $5 \mu \mathrm{m}$ could be detected, intermingling with a large amount of biological matrix matter.

Raman mapping and O-PTIR imaging would be suitable for toxicological studies of microplastics and nanoplastics ${ }^{55}$ but are challenging to apply to the quantification of microplastics and nanoplastics because the analysis speed might be too slow (Fig. $5 \mathrm{~b}, \mathrm{c}, \mathrm{d})^{56}$. The O-PTIR instrument has the potential of adopting the same automation strategy as LDIR and Raman-based particle analysis. 

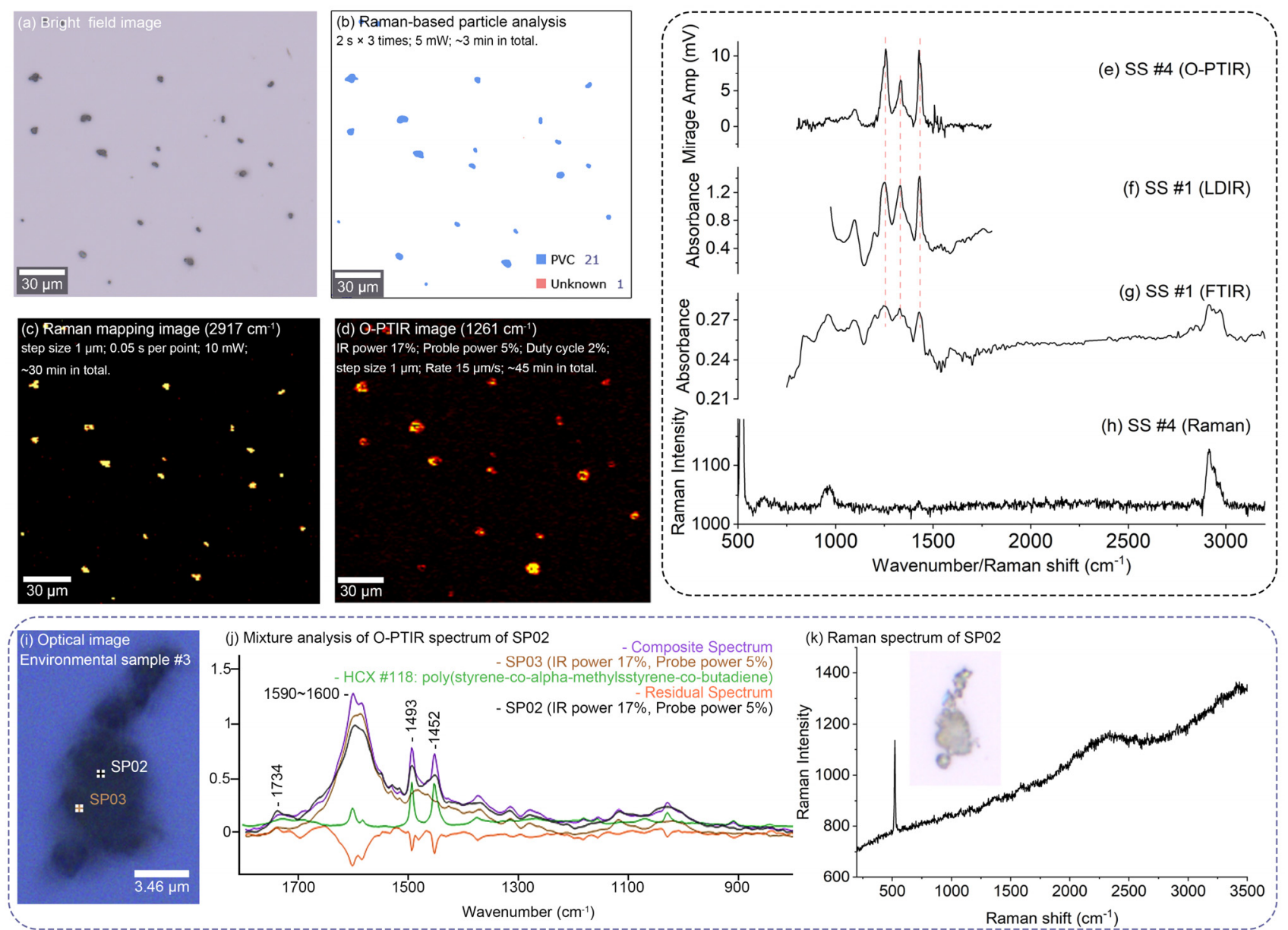

Figure 5. Material map of standard PVC particles (a) created by Raman-based particle analysis (b), Raman mapping image (c), and OPTIR image (d). IR and Raman spectra of particles (e-h, j, k). (e) and (h) were measured on the same PVC particle ( $\sim 1 \mu \mathrm{m})$ in SS\#4, but (f) and $(\mathrm{g})$ were measured on the same PVC particle $(\sim 100 \mu \mathrm{m})$ in SS\#1. (j) illustrates the result of a spectral deconvolution algorithm performed on O-PTIR spectrum "SP03" with "SP02" being a spectroscopic component in "SP03," which was obtained at the location indicated in the environmental sample (i); (k) represents the standalone Raman spectrum of the same environmental particle in the optical image.

\section{Requirement for sample preparation}

The most commonly used method for separating microplastics is filtration ${ }^{57}$. In this case, the choice of the filter membrane is very important ${ }^{58}$. According to the structure, filter membranes can be divided into multilayer/fiber-type (e.g., glass fiber, cellulose nitrate, and cellulose fiber) and monolayer-type (e.g., polycarbonate (PC), and aluminum oxide) membranes 16,57-59. Depending on the material type, filter membranes can be classified by their polymer-type (e.g., nylon, and polyethersulfone) or by inorganic (e.g., glass fiber) membranes. For the LDIR-based particle analysis, the filter membrane first needs to be sonicated in ethanol solution to extract the particles. Ethanol was further concentrated by nitrogen blowing and transferred to Kevley low-E slides. There are two sample transfer steps. It is necessary to use an inorganic single-layer filter membrane (e.g., inorganic aluminum oxide membrane) to avoid organic contamination or the potential loss of particles trapped inside the filter membrane.

FPA-FTIR imaging can make measurement of samples directly from filter membranes but requires filter membranes with as little infrared absorption as possible so the analysis algorithm can distinguish microplastic particles by IR imaging. Glass fiber membranes and polymer-type membranes are not applicable due to their highly scattering surface or their strong infrared absorption characteristics. The aluminum oxide filter has been widely used ${ }^{2,17,59-61}$ because it has no infrared absorption above $1250 \mathrm{~cm}^{-1} 59$. The authors recommended FPA-FTIR imaging with FPA to measure the transmission spectra of particles loaded on the aluminum oxide (Fig. S11). The machine learning model in Purency Microplastics Finder is trained with the transmission spectrum imaging data obtained with an aluminum oxide membrane ${ }^{33}$. The potential problem is that the largest pore size of the commercial aluminum oxide filter is only $0.2 \mu \mathrm{m}$, which could be easily blocked during the filtration process ${ }^{2}$. In addition, the particles easily gather at the edge of the filter (Fig. S11), making them indistinguishable. A silicon filter is better suited for FPA-FTIR imaging ${ }^{59}$.

Raman-based particle analysis allows the same sample preparation approach as LDIR-based particle analysis and allows the filter membrane to load particles directly. The filter membrane must be uniform and flat to meet the requirements of dark-field microscopy, confocal microscopy, and polymer analysis, which means that the filter membrane cannot be a polymer-type or fiber-type membrane. Therefore, Raman-based particle analysis is not compatible with most commercial membranes, including alumina membranes, which is not suitable for dark-field microscopy (Fig. S15). Instead, PC-coated with aluminum ${ }^{16}$ or a silicon filter ${ }^{59}$ is suitable for use in polymer analysis and dark-field microscopy. 
Particles could be loaded on the silicon wafer and goldcoated polycarbonates for the O-PTIR analysis. The technique alleviates the challenge of separating and purifying submicronsized microplastics from environmental matrices ${ }^{1}$. Microplastics might only account for a small part of the submicron-sized particles. The O-PTIR technique is very sensitive in terms of minimum detection mass, and could accurately identify environmental plastics despite the occurrence of microplastics was possibly less than $5 \%$ from all the measurement points (Fig. S14). While the analysis software (PTIR Studio) has shown excellent ease-of-use, the selection of points during the measurement process should be further automated to maximize the benefit of the instrument.

\section{CONCLUSIONS}

The answer to the question 'Are we measuring the same metrics when using these different strategies to analyze microplastics automatically?' is obviously 'No.' The number, size, and material identification results will be different among different strategies due in part to artefacts that would be present inherent to the instrumentation methods. The automated analysis of microplastics is the goal, and we believe that it will eventually replace manual analytical methods. However, a considerable amount of research must be conducted before an appropriate approach to the automated analysis of microplastics is established. In the future, comparisons among strategies, verification of strategies, and the building and sharing of the spectral database need to be better adapted. As a technological advancement, automated analysis of microplastics should be encouraged, but we need to foster the strengths and circumvent the weaknesses of different strategies.

\section{ASSOCIATED CONTENT}

\section{Supporting Information}

The Supporting Information is available free of charge on the...

The Supporting Information mainly includes the following: Detail information of standard microplastics. Photographs of environmental microplastics, a Kevley low-E slide, and a polished silicon wafer. FTIR spectra of a silicon wafer, Kevley low-E slide, and aluminum oxide filter. Material map of standard samples \#1 and \#2. Raman spectra of particles measured by 488,532 and $785 \mathrm{~nm}$ lasers. LDIR and Raman spectra of environmental particles. Raman and O-PTIR imaging of sub-micron-sized PVC particles. Time consumption and analysis speed of different strategies.

\section{AUTHOR INFORMATION}

\section{Corresponding Authors}

* Mingtan Dong - School of Environmental Studies, China University of Geosciences, Wuhan 430078, China; orcid.org/0000-0003-0303-8517; Email: hegu@cug.edu.cn

**Zejiao Luo - School of Environmental Studies, China University of Geosciences, Wuhan 430078, China; orcid.org/00000002-0894-815X; Email: zjluo@cug.edu.cn

\section{Author Contributions}

Mingtan Dong: Conceptualization, Methodology, Formal analysis, Writing - original draft

Zhenbing She: Resources, Writing - review \& editing

Xiong Xiong: Writing - review \& editing

Zejiao Luo: Resources, Funding acquisition, Writing - review \& editing

Notes

The authors declare no competing financial interest.

\section{ACKNOWLEDGMENT}

We acknowledge Agilent Technologies (China) Co., Ltd., Bruker (Beijing) Scientific Technology Co. Ltd., Quantum Design China, Purency GmbH, Photothermal Spectroscopy Corp., and WITec (Beijing) Scientific Technology Co., Ltd for providing technical support. We acknowledge Guiping Chen, Hailong $\mathrm{Hu}$, Jingjing Wang, Lukas Wander, Michael Stibi, Michael Tang, Wanghua $\mathrm{Wu}$, William Zhao, Xi Hu and Yang Gao for their assistance in this study. This research was funded by the National Key Research and Development Program (2020YFC1806804).

\section{REFERENCES}

(1) Lim, X. Nature 2021, 593, 22-25.

(2) Bergmann, M.; Mützel, S.; Primpke, S.; Tekman, M. B.; Trachsel, J.; Gerdts, G. Science Advances 2019, 5, 10.

(3) Ragusa, A.; Svelato, A.; Santacroce, C.; Catalano, P.; Notarstefano, V.; Carnevali, O.; Papa, F.; Rongioletti, M. C. A.; Baiocco, F.; Draghi, S.; D'Amore, E.; Rinaldo, D.; Matta, M.; Giorgini, E. Environ Int 2021, 146, 106274.

(4) Fred-Ahmadu, O. H.; Bhagwat, G.; Oluyoye, I.; Benson, N. U.; Ayejuyo, O. O.; Palanisami, T. Sci Total Environ 2020, 706, 135978. (5) Almeida, M.; Martins, M. A.; Soares, A. M. V.; Cuesta, A.; Oliveira, M. Environ Toxicol Pharmacol 2019, 69, 57-65.

(6) Prata, J. C.; da Costa, J. P.; Duarte, A. C.; Rocha-Santos, T. TrAC Trends in Analytical Chemistry 2019, 110, 150-159.

(7) Lenz, R.; Enders, K.; Stedmon, C. A.; Mackenzie, D. M. A.; Nielsen, T. G. Mar Pollut Bull 2015, 100, 82-91.

(8) Hitchcock, J. N.; Mitrovic, S. M. Environ Pollut 2019, 247, 457-466.

(9) Xiong, X.; Liu, Q.; Chen, X.; Wang, R.; Duan, M.; Wu, C. Chemosphere 2021, 282.

(10) Lenaker, P. L.; Baldwin, A. K.; Corsi, S. R.; Mason, S. A.; Reneau, P. C.; Scott, J. W. Environ Sci Technol 2019, 53, 1222712237.

(11) Löder, M. G. J.; Gerdts, G. In Marine Anthropogenic Litter, 2015, pp 201-227.

(12) Chae, Y.; Kim, D.; An, Y. J. Aquat Toxicol 2019, 216, 105296.

(13) Feng, J. X.; Zhao, H. S.; Gong, X. Y.; Xia, M. C.; Cai, L. S.; Yao, H.; Zhao, X.; Yan, Z. H.; Li, Z. P.; Nie, H. G.; Ma, X. X.; Zhang, S. C. Analytical Chemistry 2021, 93, 5521-5528.

(14) Laborda, F.; Trujillo, C.; Lobinski, R. Talanta 2021, 221, 121486.

(15) Li, Q.; Zeng, A.; Jiang, X.; Gu, X. J Hazard Mater 2021, 412, 125164.

(16) Ossmann, B. E.; Sarau, G.; Schmitt, S. W.; Holtmannspotter, H.; Christiansen, S. H.; Dicke, W. Anal Bioanal Chem 2017, 409, 4099-4109.

(17) da Silva, V. H.; Murphy, F.; Amigo, J. M.; Stedmon, C.; Strand, J. Analytical Chemistry 2020, 92, 13724-13733.

(18) Tagg, A. S.; Sapp, M.; Harrison, J. P.; Ojeda, J. J. Analytical Chemistry 2015, 87, 6032-6040.

(19) Weisser, J.; Beer, I.; Hufnagl, B.; Hofmann, T.; Lohninger, H.; Ivleva, N. P.; Glas, K. Water 2021, 13.

(20) Rios Mendoza, L. M.; Balcer, M. TrAC Trends in Analytical Chemistry 2019, 113, 402-408.

(21) Cowger, W.; Booth, A. M.; Hamilton, B. M.; Thaysen, C.; Primpke, S.; Munno, K.; Lusher, A. L.; Dehaut, A.; Vaz, V. P.; Liboiron, M.; Devriese, L. I.; Hermabessiere, L.; Rochman, C.; Athey, S. N.; Lynch, J. M.; De Frond, H.; Gray, A.; Jones, O. A. H.; Brander, S.; Steele, C., et al. Appl Spectrosc 2020, 3702820930292. (22) Hartmann, N. B.; Huffer, T.; Thompson, R. C.; Hassellov, M.; Verschoor, A.; Daugaard, A. E.; Rist, S.; Karlsson, T.; Brennholt, N.; Cole, M.; Herrling, M. P.; Hess, M. C.; Ivleva, N. P.; Lusher, A. L.; Wagner, M. Environ Sci Technol 2019, 53, 1039-1047.

(23) Hale, R. C.; Seeley, M. E.; La Guardia, M. J.; Mai, L.; Zeng, E. Y. Journal of Geophysical Research: Oceans 2020, 125.

(24) Olson, N. E.; Xiao, Y.; Lei, Z.; Ault, A. P. Anal Chem 2020, 92, 9932-9939.

(25) Sobhani, Z.; Al Amin, M.; Naidu, R.; Megharaj, M.; Fang, C. Anal Chim Acta 2019, 1077, 191-199. 
(26) Sobhani, Z.; Zhang, X.; Gibson, C.; Naidu, R.; Megharaj, M.; Fang, C. Water Research 2020, 174.

(27) Stock, V.; Böhmert, L.; Lisicki, E.; Block, R.; Cara-Carmona, J.; Pack, L. K.; Selb, R.; Lichtenstein, D.; Voss, L.; Henderson, C. J.; Zabinsky, E.; Sieg, H.; Braeuning, A.; Lampen, A. Archives of Toxicology 2019, 93, 1817-1833.

(28) Dong, M.; Zhang, Q.; Xing, X.; Chen, W.; She, Z.; Luo, Z. Sci Total Environ 2020, 739, 139990.

(29) Anger, P. M.; von der Esch, E.; Baumann, T.; Elsner, M.; Niessner, R.; Ivleva, N. P. TrAC Trends in Analytical Chemistry 2018, 109, 214-226.

(30) Löder, M. G. J.; Kuczera, M.; Mintenig, S.; Lorenz, C.; Gerdts, G. Environmental Chemistry 2015, 12.

(31) Simon, M.; van Alst, N.; Vollertsen, J. Water Res 2018, 142, $1-9$.

(32) Wander, L.; Vianello, A.; Vollertsen, J.; Westad, F.; Braun, U.; Paul, A. Analytical Methods 2020, 12, 781-791.

(33) Hufnagl, B.; Steiner, D.; Renner, E.; Löder, M. G. J.; Laforsch, C.; Lohninger, H. Analytical Methods 2019, 11, 2277-2285.

(34) Primpke, S.; Cross, R. K.; Mintenig, S. M.; Simon, M.; Vianello, A.; Gerdts, G.; Vollertsen, J. Appl Spectrosc 2020, 74, $1127-1138$.

(35) Reffner, J. A. 2018.

(36) Beltran, V.; Marchetti, A.; Nuyts, G.; Leeuwestein, M.; Sandt, C.; Borondics, F.; De Wael, K. Angew Chem Int Ed Engl 2021.

(37) Li, L.; Zhao, X.; Li, Z.; Song, K. J Hazard Mater 2021, 411, 124955.

(38) Scircle, A.; Cizdziel, J. V.; Tisinger, L.; Anumol, T.; Robey, D. Toxics 2020, 8 .

(39) Bassan, P.; Byrne, H. J.; Bonnier, F.; Lee, J.; Dumas, P.; Gardner, P. Analyst 2009, 134, 1586-1593.

(40) Toporski, J.; Dieing, T.; Hollricher, O. Confocal Raman Microscopy; Springer, 2018; Vol. 66.

(41) Xu, J.-L.; Lin, X.; Hugelier, S.; Herrero-Langreo, A.; Gowen, A. A. Journal of Hazardous Materials 2021, 418.

(42) Li, D.; Shi, Y.; Yang, L.; Xiao, L.; Kehoe, D. K.; Gun'ko, Y. K.; Boland, J. J.; Wang, J. J. Nature Food 2020, 1, 746-754.

(43) Munno, K.; De Frond, H.; O'Donnell, B.; Rochman, C. M. Analytical Chemistry 2020, 92, 2443-2451.

(44) Primpke, S.; Wirth, M.; Lorenz, C.; Gerdts, G. Anal Bioanal Chem 2018, 410, 5131-5141.

(45) Cowger, W.; Steinmetz, Z.; Gray, A.; Munno, K.; Lynch, J.; Hapich, H.; Primpke, S.; De Frond, H.; Rochman, C.; Herodotou, O. Anal Chem 2021, 93, 7543-7548.

(46) Fang, C.; Sobhani, Z.; Zhang, X.; Gibson, C. T.; Tang, Y.; Naidu, R. Water Res 2020, 183, 116046.

(47) Fang, C.; Sobhani, Z.; Zhang, X.; McCourt, L.; Routley, B.; Gibson, C. T.; Naidu, R. Water Res 2021, 194, 116913.

(48) Veerasingam, S.; Ranjani, M.; Venkatachalapathy, R.; Bagaev, A.; Mukhanov, V.; Litvinyuk, D.; Mugilarasan, M.; Gurumoorthi, K.; Guganathan, L.; Aboobacker, V. M.; Vethamony, P. Critical Reviews in Environmental Science and Technology 2020, 1-63.

(49) Johansen, M. P.; Cresswell, T.; Davis, J.; Howard, D. L.; Howell, N. R.; Prentice, E. Water Res 2019, 158, 392-400.

(50) Stevenson, F. J.; Goh, K. M. Geochimica et Cosmochimica Acta 1971, 35, 471-483.

(51) Knott, B. C.; Erickson, E.; Allen, M. D.; Gado, J. E.; Graham, R.; Kearns, F. L.; Pardo, I.; Topuzlu, E.; Anderson, J. J.; Austin, H. P.; Dominick, G.; Johnson, C. W.; Rorrer, N. A.; Szostkiewicz, C. J.; Copie, V.; Payne, C. M.; Woodcock, H. L.; Donohoe, B. S.; Beckham, G. T.; McGeehan, J. E. Proc Natl Acad Sci U S A 2020, 117, 25476-25485.

(52) Li, J.; Liu, H.; Paul Chen, J. Water Res 2018, 137, 362-374.

(53) Koyuncuoğlu, P.; Erden, G. Environmental Monitoring and Assessment 2021, 193, 1-28.

(54) Spadea, A.; Denbigh, J.; Lawrence, M. J.; Kansiz, M.; Gardner, P. Anal Chem 2021, 93, 3938-3950.

(55) Ramsperger, A.; Narayana, V. K. B.; Gross, W.; Mohanraj, J.; Thelakkat, M.; Greiner, A.; Schmalz, H.; Kress, H.; Laforsch, C.
Sci Adv 2020, 6 .

(56) Kappler, A.; Fischer, D.; Oberbeckmann, S.; Schernewski, G.; Labrenz, M.; Eichhorn, K. J.; Voit, B. Anal Bioanal Chem 2016, 408, 8377-8391.

(57) Cai, H.; Chen, M.; Chen, Q.; Du, F.; Liu, J.; Shi, H. Chemosphere 2020, 257.

(58) Süssmann, J.; Krause, T.; Martin, D.; Walz, E.; Greiner, R.; Rohn, S.; Fischer, E. K.; Fritsche, J. Food Control 2021, 125.

(59) Kappler, A.; Windrich, F.; Loder, M. G.; Malanin, M.; Fischer, D.; Labrenz, M.; Eichhorn, K. J.; Voit, B. Anal Bioanal Chem 2015, 407, 6791-6801.

(60) Mintenig, S. M.; Int-Veen, I.; Loder, M. G. J.; Primpke, S.; Gerdts, G. Water Res 2017, 108, 365-372.

(61) Primpke, S.; Lorenz, C.; Rascher-Friesenhausen, R.; Gerdts, G. Analytical Methods 2017, 9, 1499-1511.

\section{TABLE OF CONTENTS ARTWORK}

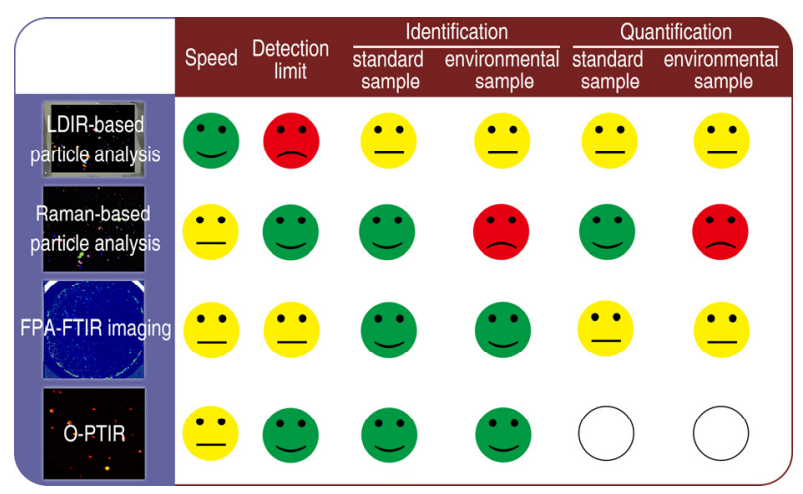

